\title{
Controlled-scattering resonances in a spherical potential well
}

\author{
${ }^{1}$ Akala, A. O. and C. Yinka-Banjo ${ }^{2}$ \\ ${ }^{1}$ Department of Physics, University of Lagos, Akoka, Yaba, Lagos, Nigeria \\ akalaovie2004@yahoo.com \\ ${ }^{2}$ Department of Computer Science, University of Lagos, Akoka, Yaba, Lagos, Nigeria \\ chikagog@yahoo.com
}

\section{ABSTRACT}

Understanding of collision processes is required in designing robust nano-particles for future applications. This study proposes a technique for controlling scattering resonances by using the tuning of well parameters to impose predetermined thresholds on resonances and bound states in collision processes. The theoretical concept of scattering in a spherical potential well, at varying depths was adopted. A scan of $q$ from 0 to $5 \pi$ at incremental steps of $q=\pi / p$ yields ( $p x$ $5)+1$ number of state(s), and $p-1$ state(s) resonate(s) at each bound state.

\section{Keywords}

Scattering, spherical potential well, resonance, collision processes

\section{INTRODUCTION}

The study of particle-particle collision processes at ultracold limit $(k \rightarrow 0)$ is fast evolving. This is because; detailed understanding of collision processes will be required in designing robust nano-particles for future applications [1,2]. At low energies, the angular momentum associated with the relative motion of the colliding particles is quantized and it is described by a set of partial waves with integer quantum number I. At sufficiently low energies, scattering is governed by quantum effects, where only contributions of first few partial waves are of essence (particularly l=0). A significant aspect of quantum scattering is the scattering resonance [3-6]. It occurs when the continuum state of two colliding particles couples to a bound state, and it is well suited for studying partial wave dynamics of collision states [7]. Scattering parameters e.g., phase shifts and cross sections change rapidly as the collision energy scans through resonance [8, 9].

The thrust of this letter is to propose a technique for controlling scattering resonances for optimum performance in future nanotechnology. The basic concept is on using tuning of well-depth and collision energy to impose pre-determined thresholds on resonances and bound states during particle-particle collision processes. In applications, external electric and magnetic fields can be used as perturbation sources to tune well parameters to predetermined thresholds on resonances and bound states.

\section{THEORY AND NUMERICAL TECHNIQUE}

Fig. 1 shows a spherical potential well and its associated parameters.

$$
\begin{aligned}
& V(r) \uparrow \quad V(r)=\left\{\begin{array}{l}
-V_{0}, r>a \\
0, r<a
\end{array}\right. \\
& \begin{array}{ll}
V_{0}(r)=A j_{l}\left(k^{\prime} a\right) & \\
R_{l}^{\prime}=\sqrt{\frac{2 m\left(E+V_{0}\right)}{\hbar^{2}}} & R_{l}(r)=B h_{l}^{(1)}(i k a) \\
\text { Ouside thle well } & k=\sqrt{\frac{-2 m E}{\hbar^{2}}}
\end{array}
\end{aligned}
$$

Fig. 1. A spherical potential well

For the radial solutions of Schrodinger equation near the origin, Bessel function of the first kind is sustained, while that of the second kind is suppressed. However, for large $r$, Hankel function of the first kind becomes valid. To sustain continuity, we need to match the solutions at the boundary, in conjunction with their first derivatives.

$$
A j_{l}(k a)=B h_{l}(i k a)
$$




$$
A k^{\prime}\left[\frac{d j_{l}\left(k^{\prime} a\right)}{d\left(k^{\prime} a\right)}\right]=B(i k)\left[\frac{d h_{l}(i k a)}{d(i k a)}\right]
$$

Furthermore, we can divide eq. (2) by (3) to obtain the boundary condition: $k \cot (k a)=-k$

The s-wave phase shift is determined as [2];

$$
\tan \delta_{0}=\frac{k \cos (k a) \sin \left(k^{\prime} a\right)-k^{\prime} \cos \left(k^{\prime} a\right) \sin (k a)}{k \sin (k a) \sin \left(k^{\prime} a\right)+k^{\prime} \cos \left(k^{\prime} a\right) \cos (k a)}
$$

We solved eq. (4) numerically, to determine the phase shifts for $s$-wave $(l=0)$ scattering of a particle of mass $m$ from a spherical well potential of depth $V_{0}=q^{2} / 2 m$ and radius $a$, where $q$ is a well depth parameter with a dimension of momentum $\left(q^{2}=k^{12}+k^{2}\right)$. We varied $V_{0}$ by scanning $q$ from 0 to $5 \pi$, at varying incremental steps.

\section{RESULTS AND DISCUSSION}

Fig 2. Shows s-wave scattering phase shift versus collision energy at varying potential well depth: (a) $q=\pi,(b) q=\pi$, (c) $q=\pi / 4$, (d) $q=\pi / 10$, (e) $q=\pi / 20$, (f) $q=\pi / 100$. As the well depth is varied for collision energies near zero, the phase shift rapidly jumps from one value of $n \pi$ to the other. The jumps are due to resonances that occur as continuum state drops into the potential well, occasioned by the variations in the well depth $[2,10]$. These jumps become sharper with increase in well-depth. The $\mathrm{n} \pi$ phase shift indicates the number of bound states $\mathrm{n}$, supported by the potential well. Each curve represents a state, and it corresponds to a different well depth separated by incremental step(s). The notable feature in figures is that phase shifts become effectively quantized in the ultra-cold limit. As $k \rightarrow \infty$, phase shift vanishes. The bunching at the bound states becomes distinctive as $k \rightarrow 0$, but becomes blurred as the well-depth separation decreases (Fig. 2f).

In Fig. $2 a$, at a separation of $q=\pi$, we have 6 curves, corresponding to $q=0, \pi, 2 \pi, 3 \pi, 4 \pi$ and $5 \pi$, with zero bound state. We have 11 curves for Fig. $2 b(q=\pi / 2)$, with only one state at the supposed position for bound states. Fig. $2 c(q=\pi / 4)$ has 21 curves, with 3 states bunching at each bound state (resonance). For Fig. $2 d(q=\pi / 10)$, there are 51 curves, with 9 states bunching at each bound state. Fig. $2 \mathrm{e}(\mathrm{q}=\pi / 20)$ has 101 curves, with 19 states bunching at each bound state, and lastly, Fig. $2 \mathrm{e}(\mathrm{q}=\pi / 100)$ has 501 curves, with 99 states bunching at each bound state. It is important to note that when a bound state is just below threshold, the scattering length is large and positive, and when the resonant state is just above threshold, the scattering length is large and negative.

Overall, as we scan $q$ from 0 to $5 \pi$ at varying incremental steps of $q=\pi / p$, the expected number of state $(s)$ is/are $(p x$ $5)+1$, with $p-1$ states resonate at each bound state. A pre-determined solid state barrier depth of $5 \pi$ for the lattice structures of a nano-material can be controlled to achieve desired resonances and bound states for the purpose of predetermined applications. Special scattering enhancers, e.g., silver nano-particles, external electric and magnetic fields can provide the needed perturbation to tune the well parameters to attain a predetermined threshold on resonances and bound states.

\section{CONCLUSIONS}

We have proposed a technique for controlling scattering resonances by using the tuning of well parameters to impose predetermined thresholds on resonances and bound states in collision processes. We scanned $q$ from 0 to $5 \pi$ at incremental steps of $q=\pi / p$ to yield $(p \times 5)+1$ number of state $(s)$, and $p-1$ states resonate at each bound state. The barrier depth of nano-material lattice structures can be internally controlled to achieve the desired resonances and bound states for purposeful applications.

\section{REFERENCES}

1. Ladd, T.D., F. Jelezko, R. Laflamme, Y. Nakamura, C. Monroe, and J. L. O’Brien, Nature Rev. 464,

45, doi:10.1038/nature08812 (2010).

2. Kendrick, B.K., J. Hazra, and N. Balakrishnan, Nature comm., 6, 7918, DOI: 10.1038/ncomms8918 (2015). Comny

3. F. Becker, S.F. and S. G. Siegel, Ann. Phys., 52, 479-485 (1969)

4. Bloch, I., Nature Phys., 1, 23, (2005).

5. Onvlee, J., A. Avoird, G. Groenenboom, and S.Y. T. van de Meerakker, J. Phys. Chem. A, DOI: 10.1021/acs.jpca.5b11951 (2016).

6. Zhu, B., J. Cooper, J. Ye, and A. M. Rey, Phys. Rev. A 94, 023612 (2016).

7. Lumer, Y., M. C. Rechtsman, Y. Plotnik, and M. Segev, Phys. Rev. A 94, 021801(R) (2016).

8. Szmytkowski, R. and C. Szmytkowski, Phys. Lett. A 235, 217-221 (1997).

9. Gensemer, S.D., R. B. Martin-Wells, A. W. Bennett, and K. Gibble, Phys. Rev. Lett. 109, 263201 (2012).

10. Hammerich, D., J. G. Muga and R. Kosloff, Israel J. Chem.29, 461-471 (1989). 
(a)

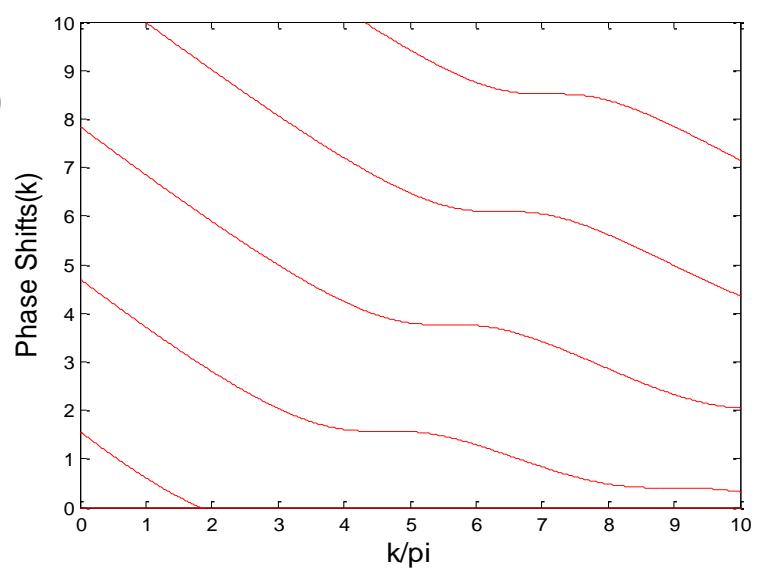

(c)

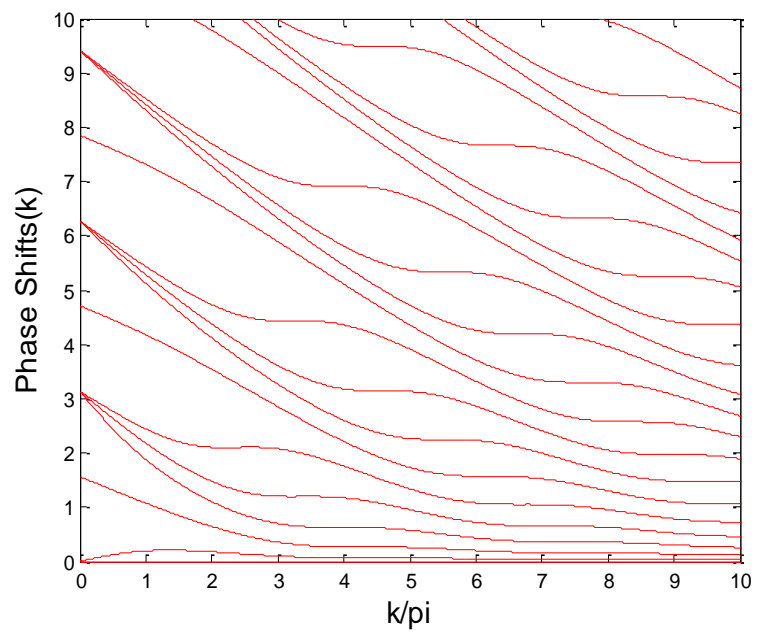

(e)

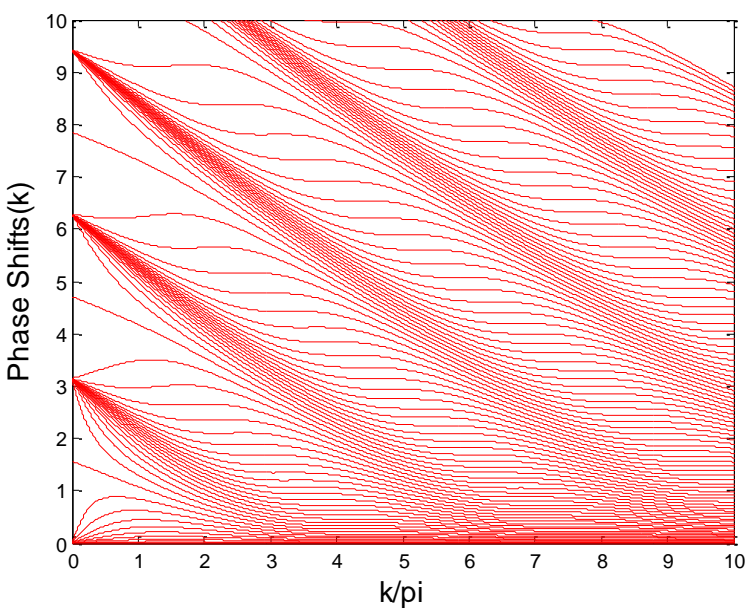

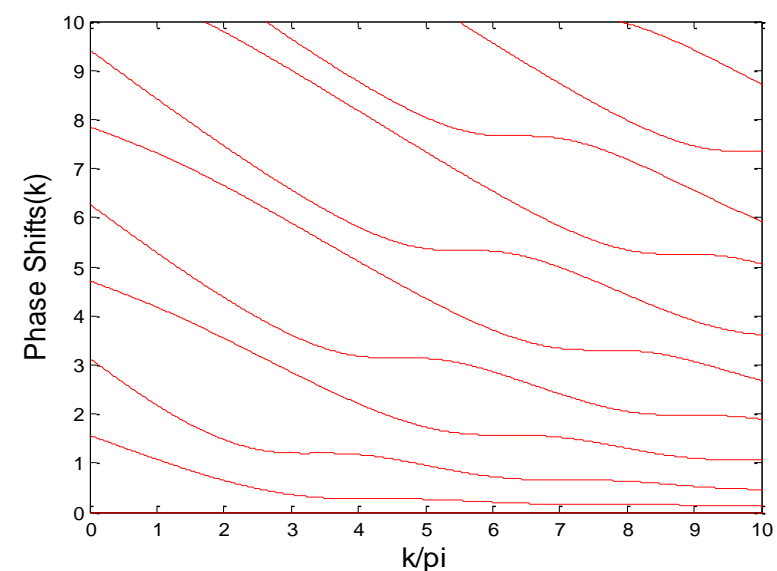

(b)

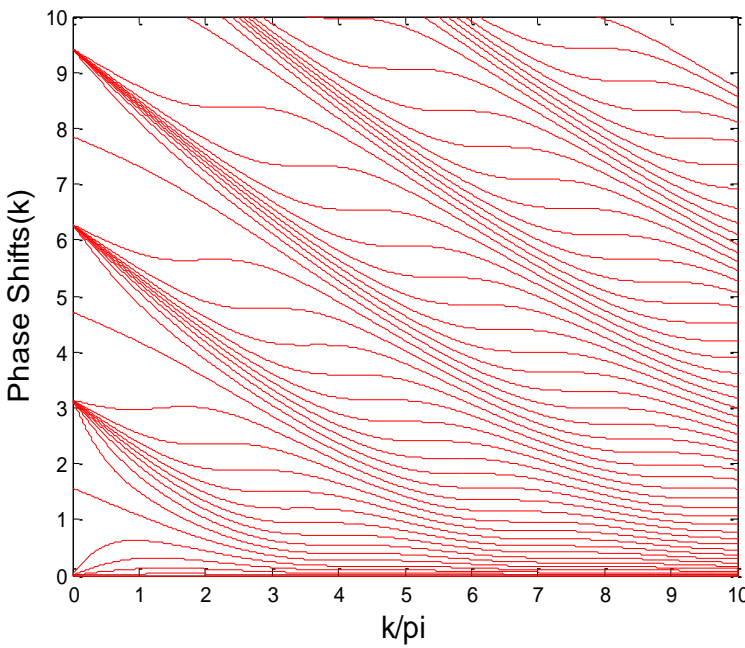

(d)

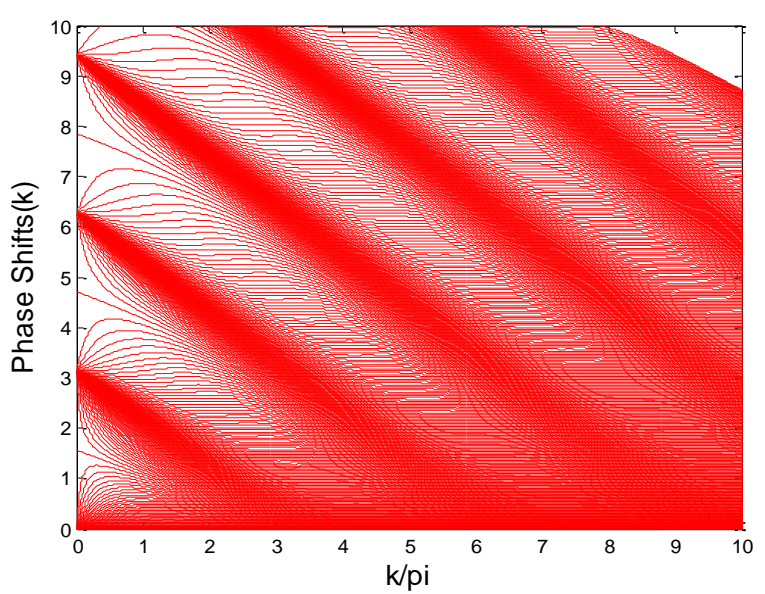

(f)

Fig 2. S-wave scattering phase shift against collision energy at varying potential well depth: (a) $q=\pi$, (b) $q=\pi$, (c) $q=\pi / 4$, (d) $q=\pi / 10$, (e) $q=\pi / 20$, (f) $q=\pi / 100$ 